\title{
JOURNAL.RU
}

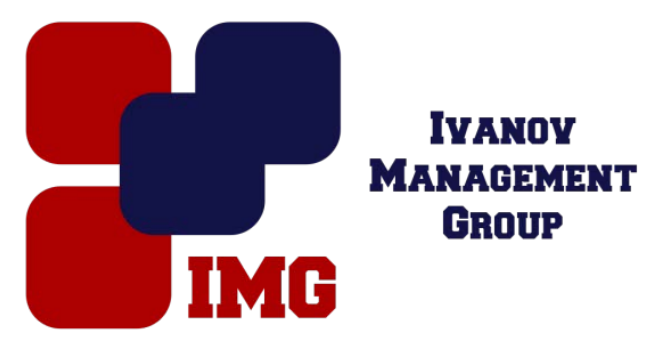

Богун В.В.

ФГБОУ ВО «ЯГПУ им. К.Д. Ушинского» Ярославль, Россия

doi: 10.18411/lj-25-12-2016-2-08

idsp 000001:lj-25-12-2016-2-08

\section{Реализация тригонометрического анализа геометрических фигур с применением принципа фундирования}

\section{Аннотация}

В данной статье рассматривается применение принципа фундирования к реализации тригонометрического анализа равнобедренных треугольников и правильных четырехугольных пирамид. Приведено понятие фундирования математических объектов, разработаны локальные и глобальная спирали фундирования применительно к исследованию рассматриваемых геометрических фигур.

Ключевые слова: принцип фундирования математических объектов, тригонометрический анализа равнобедренных треугольников и правильных четырехугольных пирамид.

\section{Abstract}

In the given paper principle application a principle of foundation mathematical objects of the trigonometrical analysis of isosceles triangles and correct quadrangular pyramids is considered. The concept фундирования mathematical plants is reduced, the local are developed and global filched фундирования with reference to research of considered geometrical figures.

Keywords: a principle of foundation mathematical objects, trigonometrical the analysis of isosceles triangles and correct quadrangular pyramids.

Принцип фундирования математических объектов заключается в поэтапном развертывании необходимых математических структур с учетом статических (признаки объектов) и динамических (выполняемые над объектами операции) свойств объектов [1]. Применительно к математике в целом [2] и тригонометрическому анализу равнобедренных треугольников и правильных честырехугольных пирамид в частности данный принцип заключается в последовательном рассмотрении математических объектов, при этом объект 
рассматриваемого уровня образован на основе одного или нескольких объектов предыдущего уровня.

В данном случае принцип фундирования заключается в последовательном переходе от рассмотрения элементарных математических задач школьного курса математики (задачи по геометрии с минимальным набором тригонометрических операций), к более сложным алгоритмическим и структурированным задачам (нахождение параметров геометрических объектов на плоскости и в пространстве с применением сложных тригонометрических структур), требующим визуального представления рассматриваемых математических объектов, явлений и процессов с точки зрения статических и динамических составляющих [3...7].

Спираль фундирования математических объектов в данном случае представляет собой последовательное усложнение реализации и визуализации определенных математических расчетов для следующих геометрических фигур:

- Равнобедренный треугольник. Используются тригонометрические функции угла при основании одного равнобедренного треугольника.

- Два равнобедренных треугольника, для которых угол при основании одного треугольника равен углу между боковыми сторонами второго. Применяются тригонометрические функции углов при основаниях двух рассматриваемых равнобедренных треугольников.

- Правильная четырехугольная пирамида. Используются тригонометрические функции углов при основаниях трех равнобедренных треугольников, образующих данную пирамиду.

- Два равнобедренных треугольника, составляющих правильную четырехугольную пирамиду. Применяются тригонометрические функции углов при основаниях двух равнобедренных треугольников, каждый из которых является одним из трех треугольников, образующих пирамиду.

Каждый уровень спирали фундирования для рассматриваемых фигур, представленный на рис. 1 ниже, включает следующие этапы при реализации и визуализации необходимых расчетов на основе получаемых значений исходных данных:

- Определение значений тригонометрических функций основных углов фигур.

- Нахождение отношений между линейными элементами фигур.

- Выявление целочисленных отношений между линейными элементами фигур.

- Нахождение пропорциональных зависимостей между линейными элементами фигур.

- Определение значений линейных элементов фигур.

- Нахождение значений координат характерных точек фигур. 
- Выявление совпадающих характерных точек фигур и определение значений координат данных точек (только при рассмотрении двух треугольников).

- Визуальное отображение геометрических фигур.

- Необходимо отметить, что каждый новый виток спирали фундирования подразумевает реализацию указанных в спирали этапов на новом уровне математических структур, базирующихся на знаниях предыдущих структур, определяя глобальную спираль фундирования, что полностью соответствует логике последовательного рассмотрения в монографии геометрических фигур.

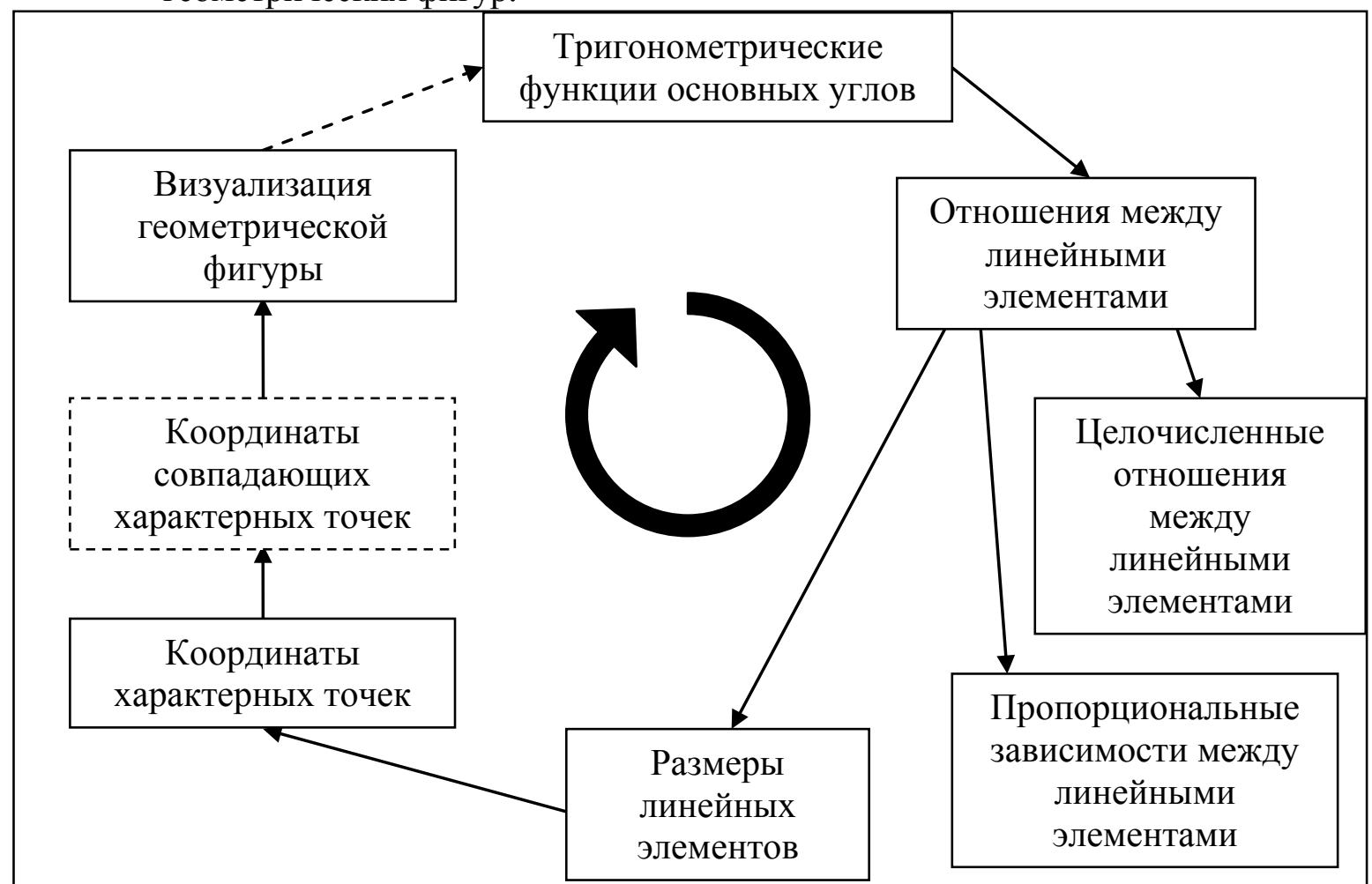

Рисунок 1. Спираль фундирования при изучении геометрических особенностей равнобедренньх треугольников и правильных четьрехугольных пирамид

Необходимо отметить, что для реализации тригонометрического анализа равнобедренных треугольников и правильных четырехугольных пирамид целесообразно применять разработанного автором программного обеспечения для графического калькулятора и персонального компьютера на локальном и сетевом уровнях [4...7] для автоматизации процесса нахождения целочисленных отношений и пропорциональных зависимостей между линейными элементами исследуемых геометрических фигур благодаря сформулированным оптимизационным поисковым алгоритмам.

Таким образом, реализацию тригонометрического анализа равнобедренных треугольника и правильных четырехугольных пирамид целесообразно осуществлять на основе концепции фундирования математических объектов с применением различных информационно- 
коммуникационных технологий (графического калькулятора и персонального компьютера на локальном и сетевом уровнях).

Работа выполнена в рамках госзадания Минобрнауки РФ

(Проект № 97)

\section{References}

1. Смирнов, Е. И. Фундирование опыта в профессиональной подготовке и инновационной деятельности педагога [Текст]: монография / Е. И. Смирнов. - Ярославль, 2012. - 646 с.

2. Пидоу, Д. Геометрия и искусство [Текст] - М.: Мир, 1979.

3. Богун, В.В. Геометрия древнего Египта [Текст] / В.В. Богун // Монография. - М.: Компания Спутник+, 2003. - 203 с.

4. Богун, В.В. Лабораторный практикум по математике с графическим калькулятором [Текст] / В.В. Богун, Е.И. Смирнов. - Ярославль: Изд-во «Канцлер», 2010. - 272 с.

5. Богун, В.В. Применение различных средств информатизации для исследования правильных четырехугольных пирамид [Текст] / В.В. Богун // Вестник Костромского государственного университета им. Н.А. Некрасова. - 2012. - № 1. - с. 106 - 110.

6. Богун, В.В. Исследование взаимосвязей между равнобедренными треугольниками с применением информационных технологий [Текст] / В.В. Богун // Ярославский педагогический вестник. - 2012. - № 4. - Том III (Естественные науки). - с. 84-96.

7. Богун, В.В. Тригонометрический анализ равнобедренных треугольников с применением информационных технологий [Текст] / В.В. Богун // Монография. - Ярославль: Изд-во «Канцлер», 2013. - 276 с. 